Chronic Obstructive Pulmonary Diseases: Journal of the COPD Foundation

\author{
Journal Club
}

\title{
Journal Club-The Intersection of Racial/Ethnic Disparities in Coronavirus Disease 2019 and COPD
}

\author{
Ron Balkissoon, MD, MSc, DIH, FRCPC ${ }^{1}$
}

\begin{abstract}
Abbreviations: coronavirus disease 2019, COVID-19; chronic obstructive pulmonary disease, COPD; COPD Genetic Epidemiology study, COPDGene ${ }^{\circledR}$; Body mass index-airway Obstruction-Dyspnea-Exercise capacity, BODE; Global initiative for chronic Obstructive Lung Disease, GOLD; International Classification of Diseases, ICD; Area Deprivation Index, ADI

Citation: Balkissoon R. Journal club: the intersection of racial/ethnic disparities in coronavirus disease 2019 and COPD. Chronic Obstr Pulm Dis. 2020;8(2):292-302. doi: https://doi.org/10.15326/jcopdf.2021.0227
\end{abstract}

1 Denver, Colorado

\section{Address correspondence to:}

Ron Balkissoon, MD, MSc, DIH, FRCPC

balkissoonr@njhealth.org

\section{Keywords}

COPD; chronic obstructive pulmonary diseases; coronavirus

disease 2019; COVID-19; racial/ethnic disparities

\section{Introduction}

The coronavirus disease 2019 (COVID-19) pandemic has highlighted that significant disparities exist for certain demographic groups in the United States with regard to the increased prevalence, morbidity and mortality related to the severe acute respiratory syndrome coronavirus-2 and the heterogenous clinical conditions referred to as COVID-19. Indeed, these disparities have been linked to long-standing systemic health and social inequities for members of certain racial and ethnic minority groups. Many investigators and commentators have suggested that the higher rates of hospitalizations and deaths seen particularly in Black and Latino communities due to COVID-19 were related to long-standing inequities in social determinants of health such as health care access and utilization, housing, education, occupation, income, wealth gaps, and discrimination. ${ }^{1-7}$ It is important to point out that smaller ethnic/racial minority communities have also been noted to face certain disparities in health and health care access. ${ }^{8,9}$
Older individuals with significant cardiovascular disease and chronic obstructive pulmonary disease (COPD) are more prone to develop more severe complications with acute respiratory distress syndrome leading to increased morbidity and mortality. Patients are more susceptible to respiratory infections that are a major cause for acute exacerbations. Perhaps not surprisingly, the same groups that have been identified to have poorer outcomes related to COVID-19 have been identified as having more severe disease and poorer outcomes if they have COPD. Indeed, the same socioeconomic factors that have been identified as the major issues that may be contributing to poorer outcomes related to COVID-19 are also the factors contributing to disparities in disease severity and outcomes for certain racial and ethnic minorities with regard to asthma and COPD. ${ }^{8,10-12}$

The recent studies that have shown reductions in COPD (and asthma) exacerbations since the pandemic seem to be more related to social isolation and use of face masks ${ }^{13-15}$ rather than any protective effect from use of inhaled corticosteroids. ${ }^{15-23}$ Interestingly, one study showed that this protective effect was seen in a large cohort of asthma patients except for Black individuals. ${ }^{14}$ Many members of these more susceptible communities have jobs and or living situations that preclude them from being able to socially distance both at work and at home. Hence, the COVID-19 pandemic has highlighted the disparities in health outcomes for many members of certain 
racial and ethnic minority communities. It gives pause to consider to what extent do we understand the degree to which such disparities impact our COPD patients who are members of these communities? In 2006, Dransfield and Bailey ${ }^{24}$ noted that while the mortality associated with COPD appeared to be increasing particularly among Black individuals, the actual underlying reasons for this remained unknown. They pointed out that while there was an abundance of published data on racial and ethnic disparities for other respiratory diseases such as asthma, this information appeared to be lacking in the COPD literature. In 2009, Shaya et $\mathrm{al}^{25}$ published a retrospective population-based cohort study using Maryland Medicaid managed care data and found that after controlling for age, gender and comorbidities, Black adults with severe asthma or coexisting COPD and severe asthma actually used fewer medical services and accounted for lower medical costs than White adults. They concluded that lower health services utilization and medical costs among Black patients with COPD and asthma may provide a possible explanation for the racial disparities in outcomes of patients with these conditions. A study by Forman and colleagues ${ }^{26}$ published in 2011 examined 2500 individuals of African and European ancestry from the COPD Genetic Epidemiology (COPDGene) ${ }^{\circledR}$ study and found that early onset COPD was seen in $42 \%(25 / 59)$ of Blacks versus $14 \%$ (45/317) of non-Hispanic Whites ( $P$ value $<0.0001)$. In 2011, Holt and colleagues examined 3.8 million COPD hospitalization records extracted from Medicare claims between 1995 and 2006 and found that there were excessive COPD-related hospitalization risks concentrated in Appalachia, the southern Great Lakes, the Mississippi Delta, the Deep South, and West Texas. ${ }^{27}$ These are areas that have significant rural communities. In 2013, Jackson and colleagues published a cross-sectional study ${ }^{28}$ of baseline data from a representative sample of patients with COPD $(\mathrm{N}=217)$ who were enrolled in a clinical trial. They noted that rural residence was associated with worse health status best represented by greater impairment of the Body mass index-airflow Obstruction-DyspneaExercise capacity (BODE) index and that rural patients had higher exposure to tobacco smoking. ${ }^{28}$

Melzer et $\mathrm{al}^{29}$ examined the hospital records of Veterans Affairs patients and looked at the outcome of any tobacco cessation medication being dispensed within 48 hours of discharge. They identified 1511 individuals of whom $16.9 \%$ were dispensed medication at discharge. In an adjusted model where several predictors were associated with decreased odds of receiving medications, Black race had an odds ratio of 0.34 ( $95 \%$ confidence interval 0.12 to 0.97 ), older age had an odds ratio of 0.96 (95\% confidence interval of 0.95 to 0.98$).^{29}$ In 2018 , Levy and colleagues ${ }^{30}$ reviewed studies that examined a link between disproportionate environmental exposures and health disparities that were noted across significant socioeconomic and racial/ethnic lines. In addition to tobacco smoke exposure, pollutants in the home, including particulate matter, nitrogen dioxide, and pesticides, were more prominent in lower socioeconomic status populations. Neighborhood crime and lack of green space also appeared to be linked to poor asthma outcomes through psychosocial pathways. It was deemed that non-modifiable individual factors such as genetic predisposition could not explain the environmental health disparities but could increase susceptibility to air pollution and other stressors. It was felt that the disparities that were noted likely stemmed from economic forces and racial/ethnic and economic segregation that have impacts on home environment, neighborhood environment, and access to healthy foods and consumer products. ${ }^{30}$

In 2018, Mamary and colleagues utilized data from the COPDGene study to evaluate the influence of race, gender, and Global initiative for chronic Obstructive Lung Disease (GOLD) ${ }^{31}$ stage on prevalence of prior COPD diagnosis at time of their enrollment into the COPDGene study. ${ }^{12}$ Data from the phase 1 cohort of 10,192 participants indicated that Blacks were less likely to have a prior COPD diagnosis versus non-Hispanic Whites regardless of the severity of their airflow obstruction that was determined at study enrollment $(P<0.0001)$. Blacks had higher odds of not having a prior COPD diagnosis at all GOLD stages of airflow obstruction. ${ }^{12}$

Further, in a study published in 2019 by Maselli and colleagues, a 10-year summary of data obtained from the COPDGene study indicated that Blacks appeared to be more likely to have an asthma/ COPD overlap condition and to have more severe disease. $^{32}$ This raises the question as to whether many Blacks who may have asthma, go undiagnosed 
and/or untreated for many years until they have significant airway remodeling and fixed airflow obstruction and are then diagnosed with COPD.

In 2019, Heintzman and colleagues published data $^{33}$ regarding a review of electronic health records in a retrospective cohort analysis of 34,849 low-income patients seen at Oregon community health centers between 2009 and 2013. They wanted to assess joint racial/ethnic and insurance disparities in diagnosis and visit rates between Latino and non-Hispanic White patients. While the overall study prevalence of obstructive lung disease was $18 \%$, Latinos demonstrated lower odds of an obstructive lung disease diagnosis (odds ratio 0.37, 95\% confidence interval 0.30 to 0.44 ). For those diagnosed prior to 2009, the uninsured (regardless of race/ethnicity) had lower visit rates during 2009 to 2013 than the insured. Health insurance was associated with basic care utilization, suggesting that lack of health insurance could lessen the quality of care for obstructive pulmonary disease in Latino and non-Hispanic White patients. ${ }^{33}$

Vaccinations for influenza have been shown to be able to significantly reduce COPD exacerbations. Saeed and colleagues ${ }^{34}$ examined pooled data from 2008 through 2016 from the Medical Expenditure Panel Survey. The presence of COPD was ascertained from self-report or International Classification of Diseases (ICD) codes. This study indicated that among adults with COPD approximately 1/3 (33.7\%; $95 \%$ confidence interval, 32.3 to 35.2 ) reported not receiving an influenza vaccine during the past year. Lack of vaccination was more frequent among adults aged 40 to 64 (44.8\%), non-Hispanic Blacks (37.7\%), Hispanics (39.7\%), those lacking a usual source of care $(52.2 \%)$, the uninsured $(63.1 \%)$, those from lower family income levels (34.6\%), and those with a lower level of completed education (35.4\%). ${ }^{34}$

In this issue of the Journal Club we review several recently published papers that revisit the extent to which there are disparities in COPD morbidity and mortality and the factors that contribute to the persistence of these differences that have been recognized for many years.

Note: Abstracts are presented in their original published format and have not been edited to match JCOPDF style.

\section{Abstract 1 \\ A Trend Analysis of COPD Mortality in the United States by Race and Sex}

Zarrabian B, Mirsaeidi M. Ann Am Thorac Soc. 2020

Dec 17. In press.

doi: https://doi.org/10.1513/AnnalsATS.202007-822OC

Rationale: Chronic respiratory diseases, wherein COPD remains the largest contributor, is the fourth leading cause of death in the USA. Updated mortality trends provide insight for targeted interventions.

Objective: To provide detailed insights into COPD mortality trends.

Methods: This study used death certificate data collected from the Center for Disease Control's Wideranging Online Data for Epidemiology Research (WONDER) system between 2004 and 2018 among Americans 40 years of age and older. We used Join point regression analysis to capture trends in annual age-adjusted COPD mortality rates and of death counts from influenza and pneumococcal disease with COPD. To place mortality trends into perspective, we examined influenza and pneumococcal vaccination rates within the same time frame using population survey data.

Results: Overall, mortality from COPD decreased, with an annual percentage change (APC) of -0.6\% (95\% CI: -0.9, -0.3) between 2004 at 72.9 per 100,000 population and 2018 at 67.4 per 100,000 population. Male COPD mortality exceeded that of females; however, male mortality continued to decline with an APC of $-1.2 \%$ (95\% CI: - 1.5, -0.9), unlike females whose death rates were overall unchanged. Further stratifying sex by race, we found that African American females were the only sociodemographic group to have had an increase in COPD mortality with an APC of $1.3 \%$ (95\% CI: $0.9,1.6)$. The number of deaths from influenza with COPD had increased over time with an observed APC of 19.58\% (95\% CI: 6.9, 33.8) between 2004 and 2018. Increased influenza mortality paralleled trends of decreased influenza vaccination rates, wherein between 2011 and 2018 there was an APC of $-5.1 \%$ (95\% CI: $-8.2,-2.0)$. This trend was also true 
for those with COPD; 451.4 per 1,000 respondents in 2011 were vaccinated against influenza compared to 352.1 per 1,000 respondents in 2018 , resulting in an APC of $-1.8 \%$ (95\% CI: -3.3, -0.2). Pneumococcal vaccination rates between 2011 and 2018 remain unchanged, meanwhile deaths from pneumococcal disease with COPD decreased; an APC of $-10.1 \%$ (95\% CI: -16.6, -3.1).

Conclusions: COPD mortality has decreased among Americans overall; however, there remain important sociodemographic groups that have not secured the same deceleration in death rates.

\section{Comments}

While it is encouraging to see there is an overall decline in COPD mortality rates in the United States, it is concerning that while the mortality rate for males continues to decline, the rate in women remains flat and this appears to be driven by the rate in Black females actually increasing. Women have been shown to have higher rates of non-smoking-related COPD, and loss of lung function with long-term tobacco use in females exceeds that of males. ${ }^{35,36}$ Furthermore, despite lower pack years of smoking, females report more severe dyspnea than males. ${ }^{37,38}$ Other studies have shown that COPD is underdiagnosed in females with fewer referrals ever made for spirometry studies. ${ }^{37,38}$ The authors offer that this may indicate that Black women have not realized the same improvements with regard to COPD management and outcomes. Black women apparently have higher odds of non-smoking COPD compared to White women, but investigators showed that this disparity could be explained secondary to education and household income. ${ }^{39}$ Eisner and colleagues found that Blacks demonstrated a greater COPD severity, but this was no longer significant when controlling for education and household income. ${ }^{40}$ Black women appear to be younger at presentation, report less pack years and began using tobacco at an older age. ${ }^{41}$ Dransfield and colleagues showed that Black females lost lung function $55 \%$ faster than White men per pack year smoked, which amounted to a $1.42 \%$ loss of lung function per pack year smoked in comparison to a $0.93 \%$ loss in White males. ${ }^{36}$

It is also instructive noting the lower vaccination rates, given the abundance of evidence of the proven efficacy of vaccinations to reduce mortality.
The authors do note certain limitations to this study including that mortality rates were extracted using death certificates with ICD-10 codes for COPD without means to validate the cause of death. The study also does not take into account comorbidities that often coexist with COPD and can contribute to overall mortality. This is particularly prescient when considering cardiovascular disease which appears to be one of the most common causes of death among COPD patients. Hence, to only extract COPD-specific mortality likely underrepresents death rates among COPD patients.

\section{Abstract 2 National Trends and Disparities in Health-Care Access and Coverage Among Adults with Asthma and COPD: 1997-2018}

Gaffney AW, Hawks L, Bore D, et al. Chest. 2021 Jan 23; In press.

doi: https://doi.org/10.1016/j.chest.2021.01.035

Background: Racial and ethnic as well as economic disparities in access to care among persons with asthma and COPD have been described, but longterm access trends are unclear.

Research question: Has health coverage and access to care and medications among adults with airways disease improved, and have disparities narrowed?

Study design and methods: Using the 1997 through 2018 National Health Interview Survey, we examined time trends in health coverage and the affordability of medical care and prescription drugs for adults with asthma and COPD, overall and by income and by race and ethnicity. We performed multivariate linear probability regressions comparing coverage and access in 2018 with that in 1997.

Results: Our sample included 76,843 adults with asthma and 30,548 adults with COPD. Among adults with asthma, lack of insurance rose in the first decade of the twenty-first century, peaking with the Great Recession, but fell after implementation of the Affordable Care Act (ACA). From 1997 through 2018, the uninsured rate among adults with asthma 
decreased from $19.4 \%$ to $9.6 \%$ (adjusted 9.27 percentage points: $95 \%$ CI, $7.1 \%-11.5 \%$ ). However, the proportions delaying or foregoing medical care because of cost or going without medications did not improve. Racial and ethnic as well as economic disparities present in 1997 persisted over the study period. Trends and disparities among those with COPD were similar, although the proportion going without needed medications worsened, rising by an adjusted 7.8 percentage points.

Interpretation: Coverage losses among persons with airways disease in the first decade of the twenty-first century were more than reversed by the ACA, but neither care affordability nor disparities improved. Further reform is needed to close these gaps.

\section{Comments}

This paper is rather sobering as far as highlighting that improvements in insurance coverage through the Affordable Care Act did not appear to translate into improved outcomes related to increased clinical evaluations or medications. While these findings certainly argue for further research and reform, it is likely that a major issue is the high co-pays and out-of-pocket costs for clinic visits, procedures, and medications. As we go forward, we need to ensure that affordable care means adequate care (coverage of diagnostic testing, services, and medications).

\section{Abstract 3 \\ Geographic Analysis of Racial Disparities in Use of Pulmonary Rehabilitation After Hospitalization for COPD Exacerbation}

Spitzer KA, Stefan MS, Priya A, et al. Chest. 2020;157(5): 1130-1137.

doi: https://doi.org/10.1016/j.chest.2019.11.044

\footnotetext{
Background: Guidelines recommend pulmonary rehabilitation (PR) after hospitalization for an exacerbation of COPD, but few patients enroll in PR. We explored whether density of PR programs explained regional variation and racial disparities in receipt of $P R$.
}

Methods: We used Centers for Medicare \& Medicaid Services data from 223,832 Medicare beneficiaries hospitalized for COPD during 2012 who were eligible for PR post-discharge. We used Hospital-Referral Regions (HRR) as the unit of analysis. For each HRR, we calculated the density of $\mathrm{PR}$ programs as a measure of program access and estimated risk-standardized rates of PR within 6 months of discharge overall, and for non-Hispanic, white, and black beneficiaries. We used linear regression to examine the relationship between access to PR and HRR PR rates. We tested for racial disparity in $\mathrm{PR}$ rates among non-Hispanic white and black beneficiaries living in the same HRRs.

Results: Across 306 HRRs, the median number of PR programs per 1,000 Medicare beneficiaries was 0.06 (interquartile range $[\mathrm{IQR}], 0.04-0.10$ ). Riskstandardized rates of $\mathrm{PR}$ ranged from $0.53 \%$ to $6.67 \%$ (median, $1.93 \%$ ). Density of PR programs was positively associated with $\mathrm{PR}$ rates overall and among non-Hispanic white beneficiaries $(P<.001)$, but this relationship was not observed among black beneficiaries. Rates were higher among non-Hispanic white beneficiaries (median, 2.08\%; IQR, 1.54\%2.87\%) compared with black beneficiaries (median, $1.19 \%$; IQR, $1.15 \%-1.20 \%)$.

Conclusions: Greater PR program density was associated with higher rates of PR for non-Hispanic white but not black beneficiaries. Further research is needed to identify reasons for this discrepancy and strategies to increase receipt of PR for black patients.

\section{Comments}

Numerous studies have demonstrated the benefits of pulmonary rehabilitation to improve health status and exercise capacity and potentially reduce readmissions in patients who have had recent exacerbations at least 2 weeks from a prior hospitalization. ${ }^{42}$ Despite the compelling evidence that shows these benefits, it remains an underutilized therapeutic intervention. ${ }^{43,44}$ There are a number of factors that could possibly explain the observed discrepancies including clinicians' unawareness of documented benefits and a lack of knowledge of how to access regional facilities and the documentation information required to enter a rehabilitation program. ${ }^{42}$ It would seem unlikely that these factors should translate into 
the observed differences between Blacks and nonHispanic Whites unless their care is significantly segregated in terms of the providers they see. In an excellent editorial of this paper, ${ }^{42}$ Criner et al suggest that there may also be patient-related factors secondary to physical limitations, psychological stress of the disease, and lack of patient motivation that can be related to a misperception that increased physical activity will not significantly improve their outcome. Prior studies of this patient cohort noted that patients who self-reported as Black or Hispanic were less likely to receive pulmonary rehabilitation within 6 months of discharge despite having more frequent COPD hospitalizations and comorbid conditions, particularly diabetes, congestive heart failure, acute myocardial infarction, cerebral vascular disease, and diabetes. ${ }^{45}$

Pulmonary rehabilitation continues to be underutilized in the broader community and it is unfortunate that it is most underutilized in a sector of the community that would likely benefit the most from the intervention.

\section{Abstract 4 Contribution of Individual and Neighborhood Factors to Racial Disparities in Respiratory Outcomes}

Ejike CO, Woo H, Galiatsatos P, et al. Am J Respir Crit Care Med. 2021;203(8):987-997.

doi: https://doi.org/10.1164/rcem.202002-0253OC

Rationale: Black adults have worse health outcomes compared with white adults in certain chronic diseases, including chronic obstructive pulmonary disease (COPD).

Objectives: To determine to what degree disadvantage by individual and neighborhood socioeconomic status (SES) may contribute to racial disparities in COPD outcomes.

Methods: Individual and neighborhood-scale sociodemographic characteristics were determined in 2,649 current or former adult smokers with and without COPD at recruitment into SPIROMICS (Subpopulations and Intermediate Outcome Measures in COPD Study).Weassessed whether racial differences in symptom, functional, and imaging outcomes (St. George's Respiratory Questionnaire, COPD
Assessment Test score, modified Medical Research Council dyspnea scale, 6-minute-walk test distance, and computed tomography [CT] scan metrics) and severe exacerbation risk were explained by individual or neighborhood SES. Using generalized linear mixed model regression, we compared respiratory outcomes by race, adjusting for confounders and individuallevel and neighborhood-level descriptors of SES both separately and sequentially.

Measurements and Main Results: After adjusting for COPD risk factors, Black participants had significantly worse respiratory symptoms and quality of life (modified Medical Research Council scale, COPD Assessment Test, and St. George's Respiratory Questionnaire), higher risk of severe exacerbations and higher percentage of emphysema, thicker airways (internal perimeter of $10 \mathrm{~mm}$ ), and more air trapping on CT metrics compared with white participants. In addition, the association between Black race and respiratory outcomes was attenuated but remained statistically significant after adjusting for individual-level SES, which explained up to $12-35 \%$ of racial disparities. Further adjustment showed that neighborhood-level SES explained another 26-54\% of the racial disparities in respiratory outcomes. Even after accounting for both individual and neighborhood SES factors, Black individuals continued to have increased severe exacerbation risk and persistently worse CT outcomes (emphysema, air trapping, and airway wall thickness).

Conclusions: Disadvantages by individual- and neighborhood-level SES each partly explain disparities in respiratory outcomes between Black individuals and white individuals. Strategies to narrow the gap in SES disadvantages may help to reduce race-related health disparities in COPD; however, further work is needed to identify additional risk factors contributing to persistent disparities.

\section{Comments}

This paper provides an interesting insight into the relative associations of personal socioeconomic status and the less often studied, neighborhood socioeconomic status, with a variety of COPD outcomes. The Subpopulations and Intermediate 
Outcome Measures in COPD Study (SPIROMICS) study had the goal of identifying new COPD subgroups and intermediate markers of disease progression. The sub-cohort (2649/2982 [88\%]) of study participants excluded patients with no history of smoking. There were 529 (20\%) who were Black (current U.S. Census Bureau data suggest that Blacks constitute $13.4 \%$ of the U.S. population). ${ }^{46}$ There were 2120 Whites in the cohort analyzed. There are some stark contrasts of individual socioeconomic comparisons between White and Black participants: The poverty rate in Whites was around $8.2 \%$ versus approximately $20.9 \%$ in Blacks $(p<0.0010)$. Approximately $14.9 \%$ of the White cohort made under 15,000 dollars compared to $40 \%$ of the Black participants $(P<0.001)$. Unfortunately, $16.4 \%$ and $22 \%$ respectively declined to answer this question which is not an insignificant number of participants. Further, $33.7 \%$ of White participants were current smokers versus $64 \%$ of the Black participants $(p<0.001)$. The neighborhood socioeconomic status was assessed using the Area Deprivation Index (ADI) national ranking score. ${ }^{47}$ The ADI is a factorbased index developed at the University of Wisconsin School of Medicine and Public Health that uses 17 U.S. Census factors reflecting poverty, education, housing and employment indicators, crime, food access, area deprivation, etc. The score is a percentile ranking that ranks neighborhoods on several factors whereby a block group with a ranking of 1 has the lowest level of disadvantage and a block group with a score of 100 would have the highest level of disadvantage. The difference in the ADI between groups in this study was substantial with an average score of 37 $( \pm 27.6)$ for Whites versus $58.6 \pm 32.8$ for Blacks $(p<0.001)$. The authors indicate that the combined individual level (12\%-35\%) and neighborhood level (26\%-54\%) factors may contribute somewhere between $33 \%-69 \%$ to the disparities in respiratory outcomes between these 2 groups. It is unfortunate that these investigators did not have data to assess the degree to which differences in access and use of health care resources contribute to the disparity. There are studies that have indeed demonstrated that moving from deprived neighborhoods can lead to improved health outcomes. ${ }^{48,49}$ The reality of the complexities of the persistent segregation of various minority communities throughout the United States illuminates the limitations of proposing this as a viable, large-scale solution. While this paper very nicely illustrates the clear impact of individual and neighborhood socioeconomic status, it points to the importance and need for further studies identifying and addressing other factors (socio-cultural beliefs, behavioral/psychological factors, stress, trauma, genetic, and biological risks) that may also influence these outcomes.

\section{The Bottom Line}

1. The COVID-19 pandemic has poignantly unveiled the persistent, significant disparities in health and health care delivery for certain racial and ethnic minority groups in the United States and elsewhere.

2. Clearly there is an overlap between being a member of certain racial/ethnic minority groups and low socioeconomic status that affects social determinants of health such as health care access and utilization, housing, education, occupation, income, wealth gaps, and perceived discrimination.

3. With the economic devastation that the COVID pandemic has had on many individuals, socioeconomic status and other social determinants of health are having an impact on a significant number of members of society. Identification and recognition of patients who face these challenges, regardless of race/ethnicity is important as many of the proposed interventions are likely relevant for all.

4. Tobacco use, exposure to indoor bio-mass fuels, and exposure to inhalant toxins, both by occupational exposure and environmental exposure related to living close to industries and other entities that have high releases of toxic inhalants, can contribute to the development and/or exacerbation of airway diseases such as asthma and COPD.

5. In order to reduce the disparities across socioeconomic strata there needs to be a number of interventions implemented including:

- improved education of the public about COPD and asthma

- improved access to affordable health care and medication coverage

- better early surveillance and detection of COPD and early intervention with preventive strategies related to such things as smoking cessation 
- reduced exposure to other environmental toxic inhalants.

- reduced health disparities for Black, Latino and other minority racial/ethnic groups as proposed by many publications. ${ }^{50-52}$ 


\section{References}

1. Van Dyke ME, Medoza MCB, Li W, et al. Racial and ethnic disparities in COVID-19 incidence by age, sex, and period among persons aged $<25$ years - 16 U.S. jurisdictions, January 1-December 31, 2020. MMWR Morb Mortal Wkly Rep. 2021;70(11):382-388. doi: https://doi.org/10.15585/mmwr.mm7011e1

2. Porter G, Desai K, George V, Coughlin S, Moore JX. Racial disparities in the epidemiology of COVID-19 in Georgia: trends since state-wide reopening. Health Equity. 2021;5(1):91-99. doi: https://doi.org/10.1089/heq.2020.0089

3. Pavlakis S, Roach ES. Follow the money: childhood health care disparities magnified by COVID-19. Pediatr Neurol. 2021;118:32-34. doi: https://doi.org/10.1016/j.pediatrneurol.2021.02.005

4. Marrett CB. Racial disparities and COVID-19: the social context. $J$ Racial Ethn Health Disparities. 2021 Mar 17; In press. doi: https://doi.org/10.1007/s40615-021-00988-8

5. Gaglioti AH, Li C, Douglas MD, et al. Population-level disparities in COVID-19: measuring the independent association of the proportion of black population on COVID-19 cases and deaths in US counties. $J$ Public Health Manag Pract. 2021;27(3):268-277. doi: https://doi.org/10.1097/PHH.0000000000001354

6. Brankston G, Merkley E, Fisman DN, et al. Socio-demographic disparities in knowledge, practices, and ability to comply with COVID-19 public health measures in Canada. Can J Public Health. 2021 Mar 24; In press.

doi: https://doi.org/10.17269/s41997-021-00501-y

7. Antwi-Amoabeng D, Beutler BD, Awad M, et al. Sociodemographic predictors of outcomes in COVID-19: examining the impact of ethnic disparities in northern Nevada. Cureus. 2021;13(2):e13128. doi: https://doi.org/10.7759/cureus.13128

8. Wen C, Liu SH, Li Y, Sheffield P, Liu B. Pediatric asthma among small racial/ethnic minority groups: an analysis of the 2006-2015 National Health Interview Survey. Public Health Rep. 2019;134(4):338-343. doi: https://doi.org/10.1177/0033354919849943

9. Thakur N, Barcelo NE, Borrell LN, et al. Perceived discrimination associated with asthma and related outcomes in minority youth: the GALA II and SAGE II studies. Chest. 2017;151(4):804-812. doi: https://doi.org/10.1016/j.chest.2016.11.027

10. Wohlford EM, Borrell LN, Elhawary JR, et al. Differential asthma odds following respiratory infection in children from three minority populations. PLoS One. 2020; 15(5):e0231782. doi: https://doi.org/10.1371/journal.pone.0231782

11. Butz AM, Bellin M, Tsoukleris M, et al. Very poorly controlled asthma in urban minority children: lessons learned. J Allergy Clin Immunol Pract. 2018;6(3):844-852. doi: https://doi.org/10.1016/j.jaip.2017.08.007
12. Mamary AJ, Stewart JI, Kinney GL, et al. Race and gender disparities are evident in COPD underdiagnoses across all severities of measured airflow obstruction. Chronic Obstr Pulm Dis. 2018;5(3):177-184. doi: https://doi.org/10.15326/jcopdf.5.3.2017.0145

13. Faria N, Costa MI, Gomes J, Sucena M. Reduction of severe exacerbations of COPD during COVID-19 pandemic in Portugal: a protective role of face masks? COPD. 2021 Mar 25; In press. doi: https://doi.org/10.1080/15412555.2021.1904387

14. Guijon OL, Morphew T, Morphew Consulting LLC, Ehwerhemuepha L, Galant S. Evaluating the impact of COVID-19 on asthma morbidity: a comprehensive analysis of potential influencing factors. Ann Allergy Asthma Immunol. 2021 Mar 24; In press. doi: https://doi.org/10.1016/j.anai.2021.03.018

15. Shah SA, Quint J, Nwaru B, Sheikh A. Impact of COVID-19 national lockdown on asthma exacerbations: interrupted time-series analysis of English primary care data. Thorax. 2021 Mar 29. In press. doi: https://doi.org/10.1136/thoraxjnl-2020-216512

16. Choi JC, Jung SY, Yoon U, et al. Inhaled corticosteroids and COVID-19 risk and mortality: a nationwide cohort study. J Clin Med. 2020;9(11):3406. doi: https://doi.org/10.3390/jcm9113406

17. Halpin DMG, Singh D, Hadfield RM. Inhaled corticosteroids and COVID-19: a systematic review and clinical perspective. Eur Respir J. 2020;55(5):2001009. doi: https://doi.org/10.1183/13993003.01009-2020

18. Maes T, Bracke K, Brusselle GG. Inhaled corticosteroids and COVID-19. Am J Respir Crit Care Med. 2020;202(6):900-902. doi: https://doi.org/10.1164/rccm.202006-2129LE

19. Maes T, Bracke K, Brusselle GG. COVID-19, asthma, and inhaled corticosteroids: another beneficial effect of inhaled corticosteroids? Am J Respir Crit Care Med. 2020;202(1):8-10.

doi: https://doi.org/10.1164/rccm.202005-1651ED

20. Rogliani P, Lauro D, Di Daniele N, Chetta A, Calzetta L. Reduced risk of COVID-19 hospitalization in asthmatic and COPD patients: a benefit of inhaled corticosteroids? Expert Rev Respir Med. 2021;15(4):561568. doi: https://doi.org/10.1080/17476348.2021.1850275

21. Schultze A, Douglas I. COVID-19 and inhaled corticosteroids-another piece in an expanding puzzle. Lancet Respir Med. 2021 Mar 4; In Press. doi: https://doi.org/10.1016/S2213-2600(21)00076-X

22. Schultze A, Walker AJ, MacKenna B, et al. Risk of COVID-19-related death among patients with chronic obstructive pulmonary disease or asthma prescribed inhaled corticosteroids: an observational cohort study using the OpenSAFELY platform. Lancet Respir Med. 2020;8(11):1106-1120. doi: https://doi.org/10.1016/S2213-2600(20)30415-X

23. Singh D., Halpin DMG. Inhaled corticosteroids and COVID-19related mortality: confounding or clarifying? Lancet Respir Med. 2020;8(11):1065-1066. doi: https://doi.org/10.1016/S2213-2600(20)30447-1 
24. Dransfield MT, Bailey WC. COPD: racial disparities in susceptibility, treatment, and outcomes. Clin Chest Med. 2006;27(3):463-471. doi: https://doi.org/10.1016/j.ccm.2006.04.005

25. Shaya FT, Maneval MS, Gbarayor CM, et al. Burden of COPD, asthma, and concomitant COPD and asthma among adults: racial disparities in a Medicaid population. Chest. 2009;136(2)405-411. doi: https://doi.org/10.1378/chest.08-2304

26. Foreman, MG, Zhang L, Murphy J, et al. Early-onset chronic obstructive pulmonary disease is associated with female sex, maternal factors, and African American race in the COPDGene study. Am J Respir Crit Care Med. 2011;184(4):414-420. doi: https://doi.org/10.1164/rccm.201011-19280C

27. Holt JB, Zhang X, Presley-Cantrell L, Croft JB. Geographic disparities in chronic obstructive pulmonary disease (COPD) hospitalization among Medicare beneficiaries in the United States. Int $J$ Chron Obstruct Pulmon Dis. 2011;6:321-328.

doi: https://doi.org/10.2147/COPD.S19945

28. Jackson BE, Coultas DB, Suzuki S, Singh KP, Bae S. Rural-urban disparities in quality of life among patients with COPD. J Rural Health. 2013;29(Suppl 1):s62-s69.

doi: https://doi.org/10.1111/jrh.12005

29. Melzer AC, Feemster LC, Collins MP, Au DH. Predictors of pharmacotherapy for tobacco use among veterans admitted for COPD: the role of disparities and tobacco control processes. $J$ Gen Intern Med. 2016;31(6):623-629.

doi: https://doi.org/10.1007/s11606-016-3623-4

30. Levy JI, Quiros-Alcala L, Fabian MP, Basra K, Hansel NN. Established and emerging environmental contributors to disparities in asthma and chronic obstructive pulmonary disease. Curr Epidemiol Rep. 2018;5(2):114-124.

doi: https://doi.org/10.1007/s40471-018-0149-9

31. Global Initiative for Chronic Obstructive Lung Disease (GOLD). Global strategy for the diagnosis, management, and prevention of chronic obstructive pulmonary disease. 2017 report. GOLD Website. Published December 2016. Accessed April 2021. https://goldcopd.org/wp-content/uploads/2017/11/GOLD-2018v6.0-FINAL-revised-20-Nov_WMS.pdf

32. Maselli DJ, Bhatt SP, Anzueto A, et al. Clinical epidemiology of COPD: insights from 10years of the COPDGenestudy. Chest. 2019;156(2):228238. doi: https://doi.org/10.1016/j.chest.2019.04.135

33. Heintzman J, Kaufmann J, Ezekial-Herrera D, et al. Asthma/COPD disparities in diagnosis and basic care utilization among low-income primary care patients. J Immigr Minor Health. 2019;21(3):659-663. doi: https://doi.org/10.1007/s10903-018-0798-2

34. Saeed GJ, Valero-Elizondo J, Mszar R, et al. Prevalence and disparities in influenza vaccination among patients with COPD in the United States. Chest. 2020;159(4):1411-1414. doi: https://doi.org/10.1016/j.chest.2020.10.058
35. Sorheim IC, Johannessen A, Gulsvik A, Bakke S, Silverman EK, DeMeo DL. Gender differences in COPD: are women more susceptible to smoking effects than men? Thorax. 2010;65(6):480-485. doi: https://doi.org/10.1136/thx.2009.122002

36. Dransfield MT, Davis JJ, Gerald LB, Bailey WC. Racial and gender differences in susceptibility to tobacco smoke among patients with chronic obstructive pulmonary disease. RespirMed. 2006;100(6):11101116. doi: https://doi.org/10.1016/j.rmed.2005.09.019

37. de Torres JP, Casanova C, Hernandez C, Abreu J, Aguirre-Jaime A, Celli BR. Gender and COPD in patients attending a pulmonary clinic. Chest. 2005;128(4):2012-2016. doi: https://doi.org/10.1378/chest.128.4.2012

38. Chapman KR, Tashkin DP, Pye DJ. Gender bias in the diagnosis of COPD. Chest. 2001;119(6):1691-1695. doi: https://doi.org/10.1378/chest.119.6.1691

39. Fuller-Thomson E, Chisholm RS, Brennenstuhl S. COPD in a population-based sample of never-smokers: interactions among sex, gender, and race. Int J Chronic Dis. 2016;5862026.

doi: https://doi.org/10.1155/2016/5862026

40. Eisner MD, Blanc PD, Omachi TA, et al. Socioeconomic status, race and COPD health outcomes. J Epidemiol Community Health 2011;65(1):26-34.

doi: https://doi.org/10.1136/jech.2009.089722

41. Chatila WM, Wynkoop WA, Vance G, Criner GJ. Smoking patterns in African Americans and whites with advanced COPD. Chest. 2004;125(1):15-21.

doi: https://doi.org/10.1378/chest.125.1.15

42. Criner GJ, Make BJ, Dorney Koppel GA. Racial disparities: another important barrier to pulmonary rehabilitation participation in patients with COPD posthospitalization. Chest. 2020;157(5):1070-1072. doi: https://doi.org/10.1016/j.chest.2020.01.029

43. Vogiatzis I, Rochester CL, Spruit MA, et al. Increasing implementation and delivery of pulmonary rehabilitation: key messages from the new ATS/ERS policy statement. Eur Respir J. 2016;47(5):1336-1341. doi: https://doi.org/10.1183/13993003.02151-2015

44. Nishi SP, Zhang W, Kuo YF, Gulshan S. Pulmonary rehabilitation utilization in older adults with chronic obstructive pulmonary disease, 2003 to 2012. J Cardiopulm Rehabil Prev. 2016;36(5):375-382. doi: https://doi.org/10.1097/HCR.0000000000000194

45. Spitzer KA, Stefan MS, Priya A, et al. Participation in pulmonary rehabilitation after hospitalization for chronic obstructive pulmonary disease among Medicare beneficiaries. Ann Am Thorac Soc. 2019;16(1):99-106.

doi: https://doi.org/10.1513/AnnalsATS.201805-332OC

46. United States Census Bureau. U.S. census data-quick facts, race and Hispanic origin. U.S. Census Bureau website. Published July 1, 2019. Accessed April 2021. https://www.census.gov/quickfacts/ fact/table/US/PST045219 
47. University of Wisconsin School of Medicine and Public Health. 2013 Area Deprivation Index. University of Wisconsin website. Published 2018. Accessed April 2021.

https://www.neighborhoodatlas.medicine.wisc.edu/

48. Ludwig J, Duncan GJ, Gennetian LA, et al. Neighborhood effects on the long-term well-being of low-income adults. Science. 2012;337(6101): 1505-1510. doi: https://doi.org/10.1126/science.1224648

49. Ludwig J, Sanbonmatsu L, Gennetian L, et al. Neighborhoods, obesity, and diabetes--a randomized social experiment. $N$ Engl J Med. 2011;365(16):1509-1519.

50. Pleasants RA, Riley IL, Mannino DM. Defining and targeting health disparities in chronic obstructive pulmonary disease. Int $J$ Chron Obstruct Pulmon Dis. 2016;11(1):2475-2496. doi: https://doi.org/10.2147/COPD.S79077

51. Louisias M, Matsui E. Disentangling the root causes of racial disparities in asthma: the role of structural racism in a 5-year-old black boy with uncontrolled asthma. J Allergy Clin Immunol Pract. 2020;8(3):11621164. doi: https://doi.org/10.1016/j.jaip.2019.12.009

52. Ali NM, Combs RM, Kakar RM, Muvuka B, Porter J. Promoting interdisciplinary, participatory approaches to address childhood asthma disparities in an urban black community. Fam Community Health. 2021;44(1)32-42.

doi: https://doi.org/10.1097/FCH.0000000000000283 